\title{
EXISTENCE THEORY FOR A TIME-DEPENDENT MEAN FIELD GAMES MODEL OF HOUSEHOLD WEALTH
}

\author{
DAVID M. AMBROSE
}

\begin{abstract}
We study a nonlinear system of partial differential equations arising in macroeconomics which utilizes a mean field approximation. This system together with the corresponding data, subject to two moment constraints, is a model for debt and wealth across a large number of similar households, and was introduced in a recent paper of Achdou, Buera, Lasry, Lions, and Moll. We introduce a relaxation of their problem, generalizing one of the moment constraints; any solution of the original model is a solution of this relaxed problem. We prove existence and uniqueness of strong solutions to the relaxed problem, under the assumption that the time horizon is small. Since these solutions are unique and since solutions of the original problem are also solutions of the relaxed problem, we conclude that if the original problem does have solutions, then such solutions must be the solutions we prove to exist. Furthermore, for some data and for sufficiently small time horizons, we are able to show that solutions of the relaxed problem are in fact not solutions of the original problem. In this way we demonstrate nonexistence of solutions for the original problem in certain cases.
\end{abstract}

\section{INTRODUCTION}

A recent paper of Achdou, Buera, Lasry, Lions, and Moll calls attention to PDE models in macroeconomics; we study a model proposed there for the distribution of wealth across many similar households [1]. In this model, the independent variables are $a$, wealth, $z$, income, and $t$, time. Each household of a given wealth and income must decide how much of their income to put towards consumption and how much to instead save. Note that wealth and savings can be positive or negative, representing debt for negative values. The authors make a mean field assumption in the modeling, so that a representative household is seen as interacting not with all the many other individual households, but only with the aggregation of these. In addition to introducing the model, the authors of [1] work with stationary solutions and state that existence and uniqueness of time-dependent solutions is an open problem. The present work gives the first theory of existence and uniqueness for time-dependent solutions.

The particular nonlinear PDE model from [1] is given by the two equations

$$
\partial_{t} v+\frac{1}{2} \sigma^{2}(z) \partial_{z z} v+\mu(z) \partial_{z} v+(z+r(t) a) \partial_{a} v+H\left(\partial_{a} v\right)-\rho v=0,
$$


(2) $\partial_{t} g-\frac{1}{2} \partial_{z z}\left(\sigma^{2}(z) g\right)+\partial_{z}(\mu(z) g)+\partial_{a}((z+r(t) a) g)+\partial_{a}\left(g H_{p}\left(\partial_{a} v\right)\right)=0$.

The dependent variables are $g$, the distribution of households, and $v$, the present discounted value of future utility derived from consumption; the discount rate is $\rho$. The nonlinear function $H$ is the Hamiltonian for the problem and is related to a given utility function, $u$; the specific form of $H$ is given below in Section 2. We consider the $z$ variable to be taken from the doman $\left[z_{\min }, z_{\max }\right]$, and the $a$ variable to be taken from $\mathbb{R}$. The function $\sigma \geq 0$ is a diffusion coefficient and the function $\mu$ is a transport coefficient. We take these to be smooth and to satisfy $\sigma\left(z_{\min }\right)=\sigma\left(z_{\max }\right)=0$ and $\mu\left(z_{\min }\right)=\mu\left(z_{\max }\right)=0$, so there is no transport or diffusion through the boundary of the domain. The interest rate $r(t)$ is not given but instead depends on the unknowns; determining $r$ will be a major focus of the present work. The model is based on models appearing previously in the economics literature [3], 8], 18].

Our choice of domain with respect to the $a$ variable is a different from [1, in which the $a$ variable was taken from the semi-infinite interval $\left[a_{\min }, \infty\right)$ for a given value $a_{\min }<0$. The theorem we prove will be for compactly supported distributions $g$, and thus our theorem is consistent with [1] with respect to the spatial domain as long as $a_{\min }$ is taken to be beyond the edge of the support of our $g$, especially at the initial time. At the end, in Section 8, we will discuss further the restriction of our solutions to the domain given in 1 .

We have two moment conditions which must be satisfied:

$$
\begin{aligned}
& \int g d a d z=1, \\
& \int a g d a d z=0 .
\end{aligned}
$$

Of course condition (3) simply expresses that $g$ is a probability measure. On the other hand (44) is an equilibrium condition which expresses that the system is closed in the sense that all money available to be borrowed in the system is in fact borrowed, and conversely all money borrowed in the system comes from within the system. Restated, condition (4) expresses that households with negative wealth have borrowed from households with positive wealth, that households with positive wealth have lent to households with negative wealth, and these total amounts borrowed and lent balance with each other. It is from the condition (44) that the interest rate, $r(t)$, is to be determined.

The equation (11) for $v$ is backward parabolic, while the equation (2) for $g$ is forward parabolic; this is the typical situation for mean field games. We therefore specify initial data $g_{0}$ for $g$, giving an initial distribution of households, and terminal data $v_{T}$ for $v$, giving a final utility function.

We actually are not able to fully solve the problem specified by (11), (2), (3), (4), with the accompanying data; rather than being a defect of our 
method, we are able to prove in some cases that this problem does not have a solution. In [1, the authors did not indicate that a general terminal condition $v_{T}$ should be specified, but instead indicated a particular choice: that $T$ should be taken to be large and that $v_{T}$ should be associated to a stationary solution of the system. We will discuss this proposed restriction on the data further in our concluding section, Section 8 below.

Another condition was stated in [1, which is related to their choice of the spatial domain with respect to the $a$ variable being $\left[a_{\min }, \infty\right)$. Since the equations (1), (2) include transport terms with respect to $a$, a boundary condition at $a=a_{\min }$ must be carefully given. This is the "state constraint boundary condition" of [1], which indicates that the relevant characteristics point into the domain; such boundary conditions for transport equations have been developed by Feller [12. The existence of the boundary at $a_{\text {min }}$ is a modeling decision, stating that lenders will no longer lend to households with debt of $a_{\min }$; the state constraint boundary condition then implies that for these households, their incomes are necessarily high enough that in the absence of further borrowing, their debt load will not increase from the accumulating interest. By considering compactly supported solutions and taking the support to be away from a given value of $a_{m i n}$, we obviate the need for any such state constraint boundary condition. Furthermore, with our compactly supported distribution $g$, our solutions feature a maximum and minimum wealth at each time, but these maximum and minimum values are not fixed in time.

The system (11), (2) is an example from the realm of mean field games, which have been introduced by Lasry and Lions [19], 20], 21], and also by Caines, Huang, and Malhame [16, [17, to study problems in game theory with a large number of similar agents. Existence theory for such systems has been developed by several authors [9], [10], [13], [14, [15], 24], [25], [26], but the system (11), (2) does not fall readily into any previously developed existence theory for two main reasons. First, some existence theory such as that of the author relies strongly on the presence of parabolic effects [5, [6], 7], but in (1), (2) the diffusion is anisotropic and cannot be used to bound derivatives with respect to the $a$ variable. Second, many of these works assume structure on the nonlinearity, especially additive separability into a part which depends on $v$ and a part which depends on $g$, and this separability is not present here. Instead, the unknowns interact through the interest rate $r(t)$, and this multiplies other terms in the equations.

The author's prior works [5], 6], [7] could be described as viewing the mean field games system as a coupled pair of nonlinear heat equations. With the anisotropic effects, we now take the view instead that (11), (2) form a coupled pair of nonlinear transport equations. Otherwise, once we have reformulated the system appropriately, the method used to prove existence and uniqueness of solutions is broadly similar to that of the author's prior work [7] this is the energy method, but adapted to the forward-backward setting of mean field games. 
The plan of the paper is as follows: in Section 2 we make some reformulation of the problem, changing to a more convenient variable than $v$. In Section 3 we take care to discuss how the interest rate $r(t)$ is calculated, introducing a modification of the original problem. In Section 4 we set up an approximation scheme for solving our modified problem. In Section 5 we prove that our approximate problems have solutions, and develop bounds for the solutions which are uniform in the approximation parameters. We pass to the limit to find solutions of our modified problem in Section 6 , to complete our existence proof. We then prove uniqueness of these solutions in Section 7. Finally, we make some concluding remarks in Section 8, including pointing out that our existence theory for the modified problem demonstrates that the original problem in some cases in fact has no solution. Our main theorems are Theorem 5 in Section 6, which establishes existence of solutions to our modified problem, and Theorem 7 in Section 7 , which establishes uniqueness of these solutions.

\section{Formulation}

We have the Hamiltonian satisfying

$$
H(p)=\max _{c \geq 0}(-c p+u(c)),
$$

where $u$ is a given consumer utility function. Since $u$ is a consumer utility function, standard economic assumptions are that $u^{\prime}(c)>0$ for all $c$ and $u^{\prime \prime}(c)<0$ for all c. For simplicity, we take $u$ to be infinitely smooth away from $c=0$, and we also assume for simplicity that the range of $u^{\prime}$ is $(0, \infty)$ and thus the domain of $\left(u^{\prime}\right)^{-1}$ is also $(0, \infty)$. We will comment briefly on the general case, in our concluding remarks in Section 8 .

Doing some calculus we see that $-c p+u(c)$ is maximized when $p=u^{\prime}(c)$, so we may rewrite $H$ as

$$
H(p)=-p\left(u^{\prime}\right)^{-1}(p)+u\left(\left(u^{\prime}\right)^{-1}(p)\right) .
$$

We may then also calculate $H_{p}$, which is given by the formula

$$
H_{p}(p)=-\left(u^{\prime}\right)^{-1}(p)-\frac{p}{u^{\prime \prime}\left(\left(u^{\prime}\right)^{-1}(p)\right)}+\frac{p}{u^{\prime \prime}\left(\left(u^{\prime}\right)^{-1}(p)\right)}=-\left(u^{\prime}\right)^{-1}(p) .
$$

Since we have taken $u$ to be smooth, we see that $H$ and $H_{p}$ inherit this smoothness.

The above calculation requires $p>0$; if instead $p \leq 0$, then there is no maximum, and the Hamiltonian would have the value $+\infty$. To restrict to $p>0$ we must take $\partial_{a} v>0$, and thus it is convenient to change variables to $w=\partial_{a} v$ and seek positive solutions for $w$. We furthermore wish to have compactly supported solutions, and this is not possible with the condition we have just stated, that $w>0$ on the whole domain. So, we introduce $y=w-f(t) w_{\infty}$ for some positive constant $w_{\infty}$, and we require $y$ to be smooth and compactly supported. We will likewise require $g$ to be compactly supported. 
We let $y=\partial_{a} v-f(t) w_{\infty}$, and seek a favorable choice of the function $f(t)$. We need to determine the equation satisfied by $y$ and also to choose our $f$. To this end, we begin by differentiating (11) with respect to $a$

$$
\begin{aligned}
\partial_{t}\left(\partial_{a} v\right) & +\frac{1}{2} \sigma^{2}(z) \partial_{z z}\left(\partial_{a} v\right)+\mu(z) \partial_{z}\left(\partial_{a} v\right) \\
& +r(t) \partial_{a} v+(z+r(t) a) \partial_{a}\left(\partial_{a} v\right)+H_{p}\left(\partial_{a} v\right) \partial_{a}\left(\partial_{a} v\right)-\rho \partial_{a} v=0 .
\end{aligned}
$$

To each $\partial_{a} v$ appearing on the right-hand side, we add and subtract $f(t) w_{\infty}$. We find the following evolution equation for $y$ :

$$
\begin{aligned}
\partial_{t} y+f^{\prime}(t) w_{\infty}+\frac{1}{2} & \sigma^{2}(z) \partial_{z z} y+\mu(z) \partial_{z} y+r(t) y+r(t) f(t) w_{\infty} \\
& +(z+r(t) a) \partial_{a} y+\Theta(y, f) \partial_{a} y-\rho y-\rho f(t) w_{\infty}=0 .
\end{aligned}
$$

Here we have introduced $\Theta$ to be the function given by

$$
\Theta(y, f)=H_{p}\left(y+f w_{\infty}\right) .
$$

We choose $f$ such that

$$
f^{\prime}(t)+r(t) f(t)-\rho f(t)=0
$$

note that this is a simple ordinary differential equation which may be solved with an integrating factor. We also must specify a terminal condition for $f$, and we take $f(T)=1$. This choice leaves the equation for $y$ as

$$
\partial_{t} y+\frac{1}{2} \sigma^{2}(z) \partial_{z z} y+\mu(z) \partial_{z} y+(r(t)-\rho) y+(z+r(t) a+\Theta(y, f)) \partial_{a} y=0
$$

In terms of $y$ and $f$, and thus also in terms of $\Theta$, our equation for $g$ is

$$
\partial_{t} g-\frac{1}{2} \partial_{z z}\left(\sigma^{2}(z) g\right)+\partial_{z}(\mu(z) g)+\partial_{a}((z+r(t) a+\Theta(y, f)) g)=0 .
$$

\section{Determining the interest Rate, ANd A Relaxed Problem}

In this section we explore the nature of the coupling between the $v$ equation (11) and the $g$ equation (2). We will proceed first in terms of $v$, and then summarize in terms of our new variable $y$. As stated in [1, the coupling is through the interest rate, $r(t)$, and this interest rate is determined through the moment condition (4).

We proceed with our first calculation on this point, which we expect is what was intended in [1]. We assume that (44) is satisfied by the data $g_{0}$.

Call $\mathcal{C}=\iint a g d a d z$. Then we differentiate $\mathcal{C}$ with respect to time:

$$
\begin{aligned}
\mathcal{C}_{t}=\iint \frac{a}{2} \partial_{z z}\left(\sigma^{2} g\right) & d a d z-\iint a \partial_{z}(\mu g) d a d z \\
& -\iint a \partial_{a}((z+r a) g) d a d z-\iint a \partial_{a}\left(H_{p} g\right) d a d z
\end{aligned}
$$


By assumptions on the diffusion and drift coefficients $\sigma$ and $\mu$, the first and second terms on the right-hand side vanish. For the third and fourth terms on the right-hand side, we integrate by parts:

$$
\begin{aligned}
\mathcal{C}_{t}=-\left.\int a(z+r a) g\right|_{a_{\text {min }}} ^{a=\infty} d z+\int & \int(z+r a) g d a d z \\
& -\left.\int a H_{p} g\right|_{a_{\min }} ^{a=\infty} d z+\iint H_{p} g d a d z .
\end{aligned}
$$

Because of our assumption of compact support with respect to $a$ in $\left(a_{m i n}, \infty\right)$, the first and third terms on the right-hand side also vanish. This leaves us with

$$
\mathcal{C}_{t}-r(t) \mathcal{C}=\mathcal{Q}
$$

with the quantity $\mathcal{Q}$ defined by $\mathcal{Q}=\iint\left(z+H_{p}\right) g$ dadz.

Unfortunately this is a difficulty, as it is unclear from this how to determine $r$ from (9). That is, if we believe that $r$ will enforce $\mathcal{C}=0$, then we must have $\mathcal{C}_{t}=0$ as well, and then (9) tells us that $\mathcal{Q}$ must equal zero as well. However this would not tell us what the interest rate is actually equal to. Worse yet, there is no reason to believe at present that $\mathcal{Q}$ would equal zero. We deal with this difficulty by generalizing the problem. Instead of seeking solutions for which $\mathcal{C}=0$, we now will determine the interest rate by insisting $\mathcal{Q}_{t}=0$.

Remark 1. Note that if $\left.g\right|_{a_{\text {min }}} \neq 0$, then there would be another term proportional to $r$ in (9). It would then be possible to choose a value of $r$ to cancel the $\mathcal{Q}$ term.

As we have just said, the condition $\mathcal{C}=0$ does indeed imply $\mathcal{Q}=0$ and thus $\mathcal{Q}_{t}=0$. Thus solutions of the original problem $(\mathcal{C}=0)$ also solve the relaxed problem $\left(\mathcal{Q}_{t}=0\right)$. In the other direction, if we have a solution of the relaxed problem, since $\mathcal{Q}_{t}=0$ we have $\mathcal{Q}=\mathcal{Q}_{0}$ for all $t$. If $\mathcal{Q}_{0}=0$ and if $\mathcal{C}(0)=0$, then we may conclude that $\mathcal{C}=0$ after all. If however $\mathcal{Q}_{0} \neq 0$ and if $\mathcal{C}(0)=0$, then we see that $\mathcal{C}_{t}(0) \neq 0$ and thus $\mathcal{C}$ is not identically zero.

We will be proving existence and uniqueness of solutions for the relaxed problem. Thus if there is a solution of the original problem, then it must be the solution we prove to exist. We will in some cases be able to guarantee that in fact $\mathcal{Q}_{0} \neq 0$, and thus in these cases, the original problem does not have a solution.

Now that we are considering the relaxed problem, we return our attention to determination of the interest rate. Taking the time derivative of $\mathcal{Q}$, we 
have

$$
\begin{aligned}
\mathcal{Q}_{t}=\iint z \partial_{t} g d a d z+\int & \int H_{p p}\left(\partial_{a} v\right)\left(\partial_{t} \partial_{a} v\right) g d a d z \\
& +\iint H_{p}\left(\partial_{a} v\right)\left(\partial_{t} g\right) d a d z:=Q_{1}+Q_{2}+Q_{3} .
\end{aligned}
$$

For each of these terms, we decompose into a part which explicitly involves $r$ and a piece which does not:

$$
\begin{aligned}
Q_{1} & =P_{1}-\iint z \partial_{a}((z+r(t) a) g) d a d z \\
Q_{2} & =P_{2}-\iint g\left(H_{p p}\left(\partial_{a} v\right)\right) \partial_{a}\left((z+r(t) a) \partial_{a} v\right) d a d z \\
Q_{3} & =P_{3}-\iint\left(H_{p}\left(\partial_{a} v\right)\right) \partial_{a}((z+r(t) a) g) d a d z
\end{aligned}
$$

where

$$
\begin{gathered}
P_{1}=-\iint z \partial_{z}(\mu(z) g) d a d z \\
P_{2}=\iint g H_{p p}\left(\partial_{a} v\right)\left(-\frac{\sigma^{2}(z)}{2} \partial_{z z}\left(\partial_{a} v\right)-\mu(z) \partial_{z}\left(\partial_{a} v\right)-H_{p}\left(\partial_{a} v\right) \partial_{a}\left(\partial_{a} v\right)+\rho \partial_{a} v\right) d a d z, \\
P_{3}=\iint H_{p}\left(\partial_{a} v\right)\left(\frac{1}{2} \partial_{z z}\left(\sigma^{2}(z) g\right)-\partial_{z}(\mu(z) g)-\partial_{a}\left(g H_{p}\left(\partial_{a} v\right)\right)\right) d a d z .
\end{gathered}
$$

We first notice that, because of the compact support with respect to $a$ in $\left(a_{\text {min }}, \infty\right)$, the integral on the right-hand side of (10) is equal to zero. We apply the derivative in the integral on the right-hand side of (11), and we integrate by parts in (12):

$$
\begin{gathered}
Q_{2}=P_{2}-r(t) \iint g\left(H_{p p}\left(\partial_{a} v\right)\right) \partial_{a} v d a d z \\
-\iint g\left(H_{p p}\left(\partial_{a} v\right)\right)(z+r(t) a) \partial_{a}^{2} v d a d z, \\
Q_{3}=P_{3}+\iint\left(H_{p p}\left(\partial_{a} v\right)\right)\left(\partial_{a}^{2} v\right)(z+r(t) a) g d a d z
\end{gathered}
$$

We introduce the notation $P=P_{1}+P_{2}+P_{3}$, and

$$
K=\iint g\left(H_{p p}\left(\partial_{a} v\right)\right) \partial_{a} v d a d z .
$$

Then adding $Q_{1}, Q_{2}$, and $Q_{3}$ back together again, we find

$$
\mathcal{Q}_{t}=P-r(t) K
$$

to arrive at this, notice that there is a cancellation when adding (13) and (14). We therefore have concluded that we may determine $r(t)$ in the relaxed problem by

$$
r(t)=\frac{P}{K}
$$


(Note that both $P$ and $K$ depend on time.)

For this to be a complete description of the determination of the interest rate, we must do two further things. First, we remark that it is clear that $K$ is nonzero. Since $H_{p}(p)=-\left(u^{\prime}\right)^{-1}(p)$ and since $u^{\prime}$ is strictly decreasing, we see that $H_{p p}(p)>0$ always. As discussed above, we are only considering solutions for which $\partial_{a} v>0$. Together with the fact that $g$ is a probability distribution, we have $K>0$. We will still, however, need to control $K$ to ensure that it cannot get arbitrarily small. Finally, we give an explicit formula for $P$, in terms of $y$ and $f$ rather than $\partial_{a} v$ :

$$
\begin{aligned}
& \text { (15) } P=P[y, f, g]=-\iint z \partial_{z}(\mu(z) g) d a d z \\
& +\iint g H_{p p}\left(y+f w_{\infty}\right)\left(-\frac{1}{2} \sigma^{2} \partial_{z z} y-\mu \partial_{z} y-H_{p}\left(y+f w_{\infty}\right) \partial_{a} y+\rho\left(y+f w_{\infty}\right)\right) d a d z \\
& +\iint H_{p}\left(y+f w_{\infty}\right)\left(\frac{1}{2} \partial_{z z}\left(\sigma^{2} g\right)-\partial_{z}(\mu g)-\partial_{a}\left(g H_{p}\left(y+f w_{\infty}\right)\right)\right) d a d z
\end{aligned}
$$

\section{ITERATIVE SCHEME}

We will prove our existence theorem using an iterative scheme, and we will now set up this scheme.

We fix $s \in \mathbb{N}$ such that $s \geq 4$; we will provide some further comments on this later. Let $A>0$ be given. We let $A_{1}=[-A, A], A_{2}=[-2 A, 2 A]$, and $A_{3}=[-3 A, 3 A]$. We let $\chi$ be such that $\chi \in C^{\infty}(\mathbb{R})$, such that $\chi(a)=1$ for $a \in A_{2}$, such that $\chi(a)=0$ for $a \in A_{3}^{c}$, and such that on each component of $A_{3} \backslash A_{2}, \chi$ is smooth and monotone. For all $a \in \mathbb{R}$, we then have $|\chi(a) a| \leq 3 A$. We will henceforth work in the spatial domain which we denote by $D$, which is $D=A_{3} \times\left[z_{\min }, z_{\max }\right]$.

Let data $g_{0} \in H^{s}(D)$ and $y_{T} \in H^{s+1}(D)$ be given, such that the support of $g_{0}$ with respect to $a$ is contained in the interior of $A_{1}$ and the support of $y_{T}$ with respect to $a$ is contained in the interior of $A_{1}$. We initialize our scheme with $g^{0}=g_{0, \delta}$ and $y^{0}=y_{T, \delta}$. Here, for small parameter values $\delta>0$, we have taken a $C^{\infty}$ function $g_{0, \delta}$ and a $C^{\infty}$ function $y_{T, \delta}$ to be within $\delta$ of $g_{0}$ in $H^{s}(D)$ and within $\delta$ of $y_{T}$ in $H^{s+1}(D)$, respectively. As we have assumed that $g_{0}$ and $y_{T}$ are each supported in the interior of $A_{1}$ with respect to the $a$ variable, we may take our approximations to also be supported in this set with respect to $a$. That our data can be approximated in this way follows from standard density results $[2$.

The solutions of our iterated system will actually depend on both $n$ and $\delta$ and would more properly be called $y^{n, \delta}$ and $g^{n, \delta}$; we will suppress this $\delta$ dependence, however, for the time being, considering for now $\delta>0$ to be fixed, and we will call the iterates $y^{n}$ and $g^{n}$, and so on. We take the function $f^{0}(t)=1$ for all $t$, and we let the initial interest rate be given as $r^{0}(t)=0$ for all $t$. We will still need to initialize $K$. 
For our constant $w_{\infty}>0$ and the data $y_{T}$ we define

$$
W=\min _{(a, z) \in D}\left(y_{T}(a, z)+w_{\infty}\right),
$$

and we require that $W>0$; this is the positivity condition for $\partial_{a} v$. Noting that our terminal data in our approximate problems is not exactly equal to $y_{T}+w_{\infty}$, we also take $\delta$ sufficiently small so that

$$
\min _{(a, z) \in D}\left(y_{T, \delta}+w_{\infty}\right) \geq \frac{3 W}{4} .
$$

We similarly define $K_{\text {data }}>0$ as

$$
K_{d a t a}=\iint g_{0}\left(H_{p p}\left(y_{T}+w_{\infty}\right)\right)\left(y_{T}+w_{\infty}\right) d a d z .
$$

Note that $K_{\text {data }}$ is positive since $g_{0}$ is a probability distribution, since $H_{p p}>$ 0 (this sign is inherited from properties of the utility function, $u$ ) and because we have taken $W>0$. We need to initialize $K$ and use something like $K_{\text {data }}$, but adapted to the data for our approximate problems,

$$
K^{0}=\iint g_{0, \delta}\left(H_{p p}\left(y_{T, \delta}+w_{\infty}\right)\left(y_{T, \delta}+w_{\infty}\right) d a d z,\right.
$$

and we may take $\delta$ sufficiently small so that

$$
K^{0} \geq \frac{3 K_{\text {data }}}{4} \text {. }
$$

Having initialized our iteration scheme with initial iterates $y^{0}=y_{T, \delta}$ and $g^{0}=g_{0, \delta}$, the support of each of $y^{0}$ and $g^{0}$ with respect to $a$ is contained in $A_{1}$ and thus also in $A_{2}$. We fix $M>1$. We may take $\delta>0$ sufficiently small so that we also have the following bounds for $y^{0}$ and $g^{0}$ :

$$
\sup _{t \in[0, T]}\left\|y^{0}(t, \cdot)\right\|_{H^{s+1}}^{2}+\left\|g^{0}(t, \cdot)\right\|_{H^{s}}^{2} \leq M\left(\left\|y_{T}\right\|_{H^{s+1}}^{2}+\left\|g_{0}\right\|_{H^{s}}^{2}\right) .
$$

These two bounds, on the supports and on the norms, are features we will seek to maintain for all subsequent iterates.

We introduce another cutoff function, related to the fact that the function $H_{p}$ is only defined for positive arguments. We have given the definition of $W>0$ above in (16). We let $\psi: \mathbb{R} \rightarrow \mathbb{R}$ be a $C^{\infty}$ function which satisfies $\psi(x)=x$ for $x \geq W / 2$, which satisfies $\psi(x)=W / 4$ for $x \leq W / 4$, and which is monotone. We define $\Theta_{c}$ by

$$
\Theta_{c}(y, f)=H_{p}\left(\psi\left(y+f w_{\infty}\right)\right) .
$$

It will be important later to note that if $y+f w_{\infty} \geq W / 2$, then $\Theta_{c}(y, f)=$ $\Theta(y, f)$.

We set up our iterative scheme, beginning with $g$ :

$$
\begin{aligned}
& \partial_{t} g^{n+1}-\frac{1}{2} \partial_{z z}\left(\sigma^{2}(z) g^{n+1}\right) \\
& +\partial_{z}\left(\mu(z) g^{n+1}\right)+\partial_{a}\left(\chi\left(z+r^{n}(t) a\right) g^{n+1}\right)+\partial_{a}\left(g^{n+1} \chi \Theta_{c}\left(y^{n}, f^{n}\right)\right)=0 .
\end{aligned}
$$


We take this with initial data

$$
g^{n+1}(0, \cdot)=g_{0, \delta} .
$$

Note that we have inserted a factor of the cutoff function $\chi$ in the transport terms. A difficulty of the system is that as long as $r \neq 0$, the transport speeds are unbounded. With the factors of $\chi$ present, this is no longer the case for our approximate equations. We will be able to remove the factors of $\chi$ by the end of our existence argument.

The transport speed in (19) with respect to the variable $a$, then, is $\chi z+$ $r^{n}(t) \chi a+\chi \Theta_{c}\left(y^{n}, f^{n}\right)$. Denote by $R$ an upper bound on $r^{n}(t)$, and denote by $Y$ and upper bound on $\Theta\left(y^{n}, f^{n}\right)$, presuming for the moment that these bounds can be found independent of our parameters $n$ and $\delta$. Then the transport speed is bounded by $z_{\max }+3 R A+Y$, independently of $n$ and $\delta$. Thus, until time $T$, the support of $g^{n+1}$ with respect to $a$, which is initially contained in $A_{1}$, remains contained in $A_{2}$ as long as $T \leq \frac{A}{z_{\max }+3 R A+Y}$.

We next give the iterated equation for $y$ :

$$
\begin{aligned}
\partial_{t} y^{n+1}+ & \frac{1}{2} \sigma^{2}(z) \partial_{z z} y^{n+1}+\mu(z) \partial_{z} y^{n+1}+r^{n}(t) y^{n+1} \\
& +\left(\chi z+r^{n}(t) \chi a\right) \partial_{a} y^{n+1}+\chi \Theta_{c}\left(y^{n}, f^{n}\right) \partial_{a} y^{n+1}-\rho y^{n+1}=0 .
\end{aligned}
$$

As above, we take this with mollified data

$$
y^{n+1}(T, \cdot)=y_{T, \delta} .
$$

Again, the solutions may more properly be called $y^{n, \delta}$, but we will suppress the $\delta$ dependence for the time being. Note that we have the same transport speed with respect to $a$ as in the $g^{n+1}$ equation, and therefore we have the same support properties; with initial data supported in $A_{1}$, and with the presumed upper bounds, the support of $y^{n+1}$ remains in $A_{2}$ as long as $T \leq \frac{A}{z_{\max }+3 R A+Y}$.

To finish specifying the iterated problem, we must specify $f^{n+1}$ and $r^{n+1}$, and the latter of these will require specifying $P^{n+1}$ and $K^{n+1}$. We take $f^{n+1}$ to be the solution of the ordinary differential equation

$$
\left(f^{n+1}\right)^{\prime}(t)+r^{n}(t) f^{n+1}(t)-\rho f^{n+1}(t)=0,
$$

with terminal condition $f(T)=1$. Notice that the solution of this terminal value problem is

$$
f^{n+1}(t)=\exp \left\{\int_{t}^{T} r^{n}\left(t^{\prime}\right)-\rho d t^{\prime}\right\}
$$

We take $r^{n+1}$ to be given by

$$
r^{n+1}=\frac{P^{n}}{K^{n}}
$$


where we need to define $P^{n}$ and $K^{n}$. Consistent with our previous definition of $K$ we denote

$$
K[y, g, f]=\iint g\left(H_{p p}\left(y+f w_{\infty}\right)\right)\left(y+f w_{\infty}\right) d a d z ;
$$

but $K\left[y^{n}, g^{n}, f^{n}\right]$ is not sufficient for use in our iterative scheme because we need to use the cutoff function $\psi$. Thus, for any value of $n$, given $y^{n}, f^{n}$, and $g^{n}$, we define $K^{n+1}$ as

$$
K^{n+1}=\iint g^{n}\left(H_{p p}\left(\psi\left(y^{n}+f^{n} w_{\infty}\right)\right)\right)\left(y^{n}+f^{n} w_{\infty}\right) d a d z .
$$

Finally, recalling $P[y, f, g]$ as defined in (15), we must introduce a version $P_{c}$ which involves the cutoff function $\psi$ :

$$
\begin{aligned}
& P_{c}[y, f, g]=-\iint z \partial_{z}(\mu(z) g) d a d z \\
&+\iint g H_{p p}\left(\psi\left(y+f w_{\infty}\right)\right)\left(-\frac{1}{2} \sigma^{2} \partial_{z z} y-\mu \partial_{z} y-\Theta_{c}(y, f) \partial_{a} y+\rho\left(y+f w_{\infty}\right)\right) d a d z \\
&+\iint \Theta_{c}(y, f)\left(\frac{1}{2} \partial_{z z}\left(\sigma^{2} g\right)-\partial_{z}(\mu g)-\partial_{a}\left(g \Theta_{c}(y, f)\right)\right) d a d z .
\end{aligned}
$$

We can then define our iterated $P$ as

$$
P^{n}=P_{c}\left[y^{n}, f^{n}, g^{n}\right]
$$

\section{EXISTENCE AND BOUNDS FOR THE ITERATES}

In order to eliminate our approximation parameters, i.e. send $n \rightarrow \infty$ and $\delta \rightarrow 0$, we need to establish bounds for the iterates which are uniform with respect to $n$ and $\delta$. We fix a value $M>1$ and we assume the following are satisfied by the $n$-th iterates:

$$
\begin{gathered}
\left\|y^{n}\right\|_{H^{s+1}} \leq M\left\|y_{T}\right\|_{H^{s+1}}, \\
\left\|g^{n}\right\|_{H^{s}} \leq M\left\|g_{0}\right\|_{H^{s}}, \\
f^{n} \in\left[\frac{1}{2}, 2\right], \quad \forall t \in[0, T], \\
K^{n} \geq \frac{K_{\text {data }}}{2}, \quad \forall t \in[0, T] .
\end{gathered}
$$

We furthermore assume that the $n$-th iterates are infinitely smooth.

Based on these values, we define a value $P_{\max }$; we take this to be the supremum of the set of values $\left\{\left|P_{c}[\tilde{y}, \tilde{f}, \tilde{g}]\right|\right\}$, where $\tilde{y}, \tilde{g}$, and $\tilde{f}$ satisfy

$$
\|\tilde{y}\|_{H^{s+1}} \leq M\left\|y_{T}\right\|_{H^{s+1}}, \quad\|\tilde{g}\|_{H^{s}} \leq M\left\|g_{0}\right\|_{H^{s}}, \quad \tilde{f} \in[1 / 2,2] .
$$


With this definition, we then have our inductive hypothesis for the iterates for the interest rate:

$$
r^{n} \in\left[-\frac{2 P_{\max }}{K_{\text {data }}}, \frac{2 P_{\max }}{K_{\text {data }}}\right], \quad \forall t \in[0, T] .
$$

Finally, we have one more condition we wish to have satisfied for our iterates, and that is the positivity condition for $\partial_{a} v$. Recall the definition of $W>0$ in (16). Then we desire that the following condition is satisfied for $y^{n}$ and $f^{n}$ :

$$
\min _{(t, a, z) \in[0, T] \times D}\left(y^{n}(t, a, z)+f^{n}(t) w_{\infty}\right) \geq \frac{W}{2} .
$$

Note that with our specification of the initial iterates, the bounds (27), (28), (29), and (31) are satisfied for $n=0$. By (17) we have satisfied (32) as well for $n=0$. Similarly, by (18), we have satisfied (30) when $n=0$. We may also note that all of the initial iterates are in $C^{\infty}$. We must verify that each of (27), (28) , (29), (30), (31), and (32) are satisfied for the $(n+1)$-st iterates, but first we must ensure that the $(n+1)$-st iterates exist.

Lemma 2. Let $T>0$, and let $y^{n}, g^{n}, r^{n}, f^{n}$, and $K^{n}$ be as described above, on the time interval $[0, T]$. There exists a unique $C^{\infty}$ solution $g^{n+1}$ to the initial value problem (19), (20) on the time interval $[0, T]$.

Proof. We prove existence by the energy method, the steps of which are to introduce mollifiers, use the Picard theorem to get existence of solutions, prove an estimate uniform with respect to the mollification parameter, and then pass to the limit as the mollification parameter vanishes. To use standard theory of mollifiers, we first replace our spatial domain with a torus.

We make an extension of the domain in the $z$ variable. Let $\omega \in \mathbb{N}$ be any finite degree of regularity, sufficiently large. We take $\widetilde{\sigma}, \widetilde{\mu}$, and $\widetilde{\Theta}$ to be $H^{\omega+2}$ extensions of $\sigma, \mu$, and $\Theta\left(y^{n}, f^{n}\right)$ to the domain $\left[z_{\min }-3, z_{\max }+3\right]$ (for $\sigma$ and $\mu$ ) and to the domain $A_{3} \times\left[z_{\min }-3, z_{\max }+3\right]$ (for $\Theta$ ). There are many versions of the existence of such extensions available in the literature, and we cite 23 in particular. We let $\phi$ be a cutoff function which is equal to 1 for $z \in\left[z_{\min }-1, z_{\max }+1\right]$ and which is equal to zero on $\left[z_{\min }-\right.$ $\left.3, z_{\min }-2\right]$ and on $\left[z_{\max }+2, z_{\max }+3\right]$, and which is smooth and monotone on the remaining components of the new $z$ domain. In writing an evolution equation to approximate (19), we will replace $\sigma, \mu$, and $\Theta\left(y^{n}, f^{n}\right)$ with $\phi \widetilde{\sigma}$, $\phi \widetilde{\mu}$, and $\phi \widetilde{\Theta}$, respectively. We also replace the transport coefficient $\chi\left(z+r^{n} a\right)$ with $\phi \chi\left(z+r^{n} a\right)$. We take $\widetilde{g}_{0}$ to be an $H^{\omega}$ extension of $g_{0, \delta}$, and we will use data $\phi \chi \widetilde{g}_{0}$.

The coefficients in our new evolution equation, because they are zeroed out at the ends of the interval $\left[z_{\min }-3, z_{\max }+3\right]$, are periodic with respect to $z$. Similarly, the coefficients are all also periodic with respect to $a$ on $A_{3}$. Because of the presence of $\chi$ and $\phi$ in our proposed data, we also have periodic initial data. We call our new domain $\widetilde{D}$, and we consider this now to be a torus, i.e. we take periodic boundary conditions. We let $\mathcal{J}_{\tau}$ be a 
standard mollifier on the two-dimensional torus with parameter $\tau>0$. We introduce an approximate equation:

$$
\begin{aligned}
& \partial_{t} h^{\tau}-\frac{1}{2} \partial_{z z} \mathcal{J}_{\tau}\left((\phi \widetilde{\sigma})^{2} \mathcal{J}_{\tau} h^{\tau}\right) \\
+ & \partial_{z} \mathcal{J}_{\tau}\left((\phi \widetilde{\mu}) \mathcal{J}_{\tau} h^{\tau}\right)+\partial_{a} \mathcal{J}_{\tau}\left(\phi \chi\left(z+r^{n} a\right) \mathcal{J}_{\tau} h^{\tau}\right)+\partial_{a} \mathcal{J}_{\tau}\left(\phi \chi \widetilde{\Theta} \mathcal{J}_{\tau} h^{\tau}\right)=0 .
\end{aligned}
$$

As we have said, we take this evolution with initial condition

$$
h(0, \cdot)=\phi \chi \widetilde{g}_{0} .
$$

The presence of the mollifiers turns all derivatives on the right-hand side of (33) into bounded operators; the Picard Theorem [22] then implies that there exists a solution for a time $T_{\tau}>0$. This solution may be continued as long as the solution does not blow up; in this case, an energy estimate, using standard mollifier properties and integration by parts, implies that the $H^{\omega}(\widetilde{D})$ norm of $h$ does not blow up on $[0, T]$. We introduce an energy, equivalent to the square of the $H^{\omega}(\widetilde{D})$ norm,

$$
E(t)=\sum_{j=0}^{\omega} \sum_{\ell=0}^{\omega-j} E_{j, \ell}(t), \quad E_{j, \ell}(t)=\frac{1}{2} \int_{\widetilde{D}}\left(\partial_{a}^{j} \partial_{z}^{\ell} h^{\tau}(t, a, z)\right)^{2} d a d z .
$$

Taking the time derivative of the energy, using the facts that $\mathcal{J}_{\tau}$ commutes with derivatives and is self-adjoint, and using other mollifier properties such as $\left\|\mathcal{J}_{\tau} f\right\|_{H^{m}} \leq\|f\|_{H^{m}}$ for any $f$ and any $m$, and integrating by parts yields the conclusion

$$
\frac{d E}{d t} \leq c E
$$

where $c$ is independent of $\tau$. (We do not provide further details of this energy estimate as it is very similar to the estimate in Theorem 4 below). The bound (34) implies that the solutions $h^{\tau}$ are uniformly bounded in $H^{\omega}(\widetilde{D})$ with respect to the approximation parameter $\tau$, and that our solutions $h^{\tau}$ all exist on the common time interval $[0, T]$.

The uniform bound implies that the first derivatives of the solutions with respect to $a, z$, and $t$ are all uniformly bounded, and thus our solutions $h^{\tau}$ form an equicontinuous family. Thus there is a uniformly convergent subsequence (which we do not relabel), as $\tau$ vanishes; we call the limit $h$. Uniform convergence implies convergence in $L^{2}$ in a bounded domain, so we see that $h^{\tau}$ converges to $h$ in $C\left([0, T] ; L^{2}(\widetilde{D})\right)$. Using the uniform bound in $H^{\omega}(\widetilde{D})$, a standard Sobolev interpolation theorem (see [4], for example) then implies convergence in $C\left([0, T] ; H^{\omega-1}(\widetilde{D})\right)$. Furthermore the uniform bound implies that we have a weak limit at every time in $H^{\omega}$, and this weak limit must be $h$, so we have $h \in L^{\infty}\left([0, T] ; H^{\omega}\right)$ as well.

Taking the integral with respect to time of (33) and then passing to the limit as $\tau$ vanishes (this is possible because of the regularity we have established, including convergence in $\left.C\left([0, T] ; H^{\omega-1}\right)\right)$, and then differentiating 
with respect to time, we see that $h$ satisfies

$$
\partial_{t} h-\frac{1}{2} \partial_{z z}\left((\phi \widetilde{\sigma})^{2} h\right)+\partial_{z}(\phi \widetilde{\mu} h)+\partial_{a}\left(\phi \chi\left(z+r^{n} a\right) h\right)+\partial_{a}(\phi \chi \widetilde{\Theta} h)=0 .
$$

When taking this limit we again use various standard mollifier properties; a good list of such properties can be found in Lemma 3.5 of [22. Perhaps the most useful of these to arrive at (35) is, for any $m \in \mathbb{N}$,

$$
\left\|\mathcal{J}_{\tau} f-f\right\|_{H^{m}} \leq \tau\|f\|_{H^{m+1}} .
$$

We define $g^{n+1}$ to be the restriction of $h$ to the domain $D$. On $D$, we have $\phi=1, \widetilde{\sigma}=\sigma, \widetilde{\mu}=\mu, \widetilde{\Theta}=\Theta\left(y^{n}, f^{n}\right)$, and $\widetilde{g}_{0}=g_{0, \delta}$. Furthermore on $D$ we also have $\chi g_{0, \delta}=g_{0, \delta}$. We conclude that $g^{n+1}$ satisfies (19) and (20).

We have two further points to make, to complete the proof. First, we mention that uniqueness of solutions of the initial value problem (19), (20) is straightforward. The initial value problem satisfied by the difference of two solutions is a linear equation with zero forcing and zero data, and an estimate in $L^{2}$ for the difference of two smooth solutions can be made. Finally, on regularity, we mention that the regularity parameter $\omega$ was arbitrary, so we see that the solution $g^{n+1}$ is infinitely smooth with respect to the spatial variables. Upon taking higher derivatives of (19) with respect to time, it can be seen that the solutions are also infinitely smooth with respect to time. This completes the proof.

We also have existence of the iterated $y^{n+1}$, given in the following lemma.

Lemma 3. Let $y^{n}, g^{n}, r^{n}, f^{n}$, and $K^{n}$ be as described above. There exists a unique $C^{\infty}$ solution $y^{n+1}$ to the initial value problem (21), (22) on the time interval $[0, T]$.

We omit the proof of Lemma 3, as the method is entirely the same as that of Lemma 2 .

To conclude this section, we mention that it is immediate from their definitions and the smoothness assumptions on the $n$-th iterates that $f^{n+1}$, $K^{n+1}$, and $r^{n+1}$ are $C^{\infty}$ in time.

5.1. Uniform Bounds. Recall that we have fixed $s \in \mathbb{N}$ satisfying $s \geq$ 4, and we have taken $g_{0} \in H^{s}$ and $y_{T} \in H^{s+1}$. The requirement $s \geq 4$ will guarantee that the solutions we find are classical solutions of the PDE system, and will allow us to use Sobolev embedding and related inequalities as needed. Note that while we have demonstrated above that the iterates are infinitely smooth, this has relied on the $C^{\infty}$ approximation $g_{0, \delta}$ to the intended data $g_{0}$; with the data $g_{0} \in H^{s}$ and $y_{T} \in H^{s+1}$, we can only expect bounds on the iterates which are uniform with respect to the parameters in these spaces.

Theorem 4. There exists $T_{*}>0$ such that if the time horizon satisfies $T \in$ $\left(0, T_{*}\right)$, then for all $n \in \mathbb{N}$ and for all $\delta>0$, the iterates $\left(y^{n}, g^{n}, f^{n}, K^{n}, r^{n}\right)$ defined above satisfy (27), (28), (29), (301), (31), and (32). 
Proof. The proof will be by induction. We have remarked previously that (27), (28), (29), (30), (31), and (32) hold in the case $n=0$; this is the base case. The statements (27), (28), (29), (30), (31), and (32) then together constitute the inductive hypothesis.

We begin by determining a bound for the next iterate $g^{n+1}$. We let the functional $E_{j, \ell}$ be given by

$$
E_{j, \ell}(t)=\frac{1}{2} \int_{D}\left(\partial_{a}^{j} \partial_{z}^{\ell} g^{n+1}\right)^{2} d a d z,
$$

and we sum over $j$ and $\ell$ to form the energy $E(t)$,

$$
E(t)=\sum_{j=0}^{s} \sum_{\ell=0}^{s-j} E_{j, \ell}(t) .
$$

Of course, the energy $E$ is equivalent to the square of the $H^{s}$-norm of $g^{n+1}$.

We will now demonstrate a bound for the growth of the energy. For given values of $j$ and $\ell$, we take the time derivative of $E_{j, \ell}$ :

$$
\frac{d E_{j, \ell}}{d t}=\int_{D}\left(\partial_{a}^{j} \partial_{z}^{\ell} g^{n+1}\right)\left(\partial_{a}^{j} \partial_{z}^{\ell} \partial_{t} g^{n+1}\right) d a d z .
$$

We therefore need to write a helpful expression for $\partial_{a}^{j} \partial_{z}^{\ell} \partial_{t} g^{n+1}$. Applying derivatives to (19), we arrive at the expression

$$
\begin{aligned}
\partial_{a}^{j} \partial_{z}^{\ell} \partial_{t} g^{n+1}= & \frac{1}{2} \sigma^{2} \partial_{a}^{j} \partial_{z}^{\ell+2} g^{n+1}+(\ell+2) \sigma\left(\partial_{z} \sigma\right) \partial_{a}^{j} \partial_{z}^{\ell+1} g^{n+1}-\mu \partial_{a}^{j} \partial_{z}^{\ell+1} g^{n+1} \\
& -\left(\chi z+r^{n} \chi a\right) \partial_{a}^{j+1} \partial_{z}^{\ell} g^{n+1}-\chi \Theta_{c}\left(y^{n}, f^{n}\right) \partial_{a}^{j+1} \partial_{z}^{\ell} g^{n+1}+\Phi
\end{aligned}
$$

where $\Phi$ is a collection of terms which will be more routine to estimate. We can write $\Phi$ explicitly:

$$
\begin{gathered}
\Phi=\frac{1}{2} \sum_{m=2}^{\ell}\left(\begin{array}{c}
\ell+2 \\
m
\end{array}\right)\left(\partial_{z}^{m} \sigma^{2}\right) \partial_{a}^{j} \partial_{z}^{\ell+2-m} g^{n+1}-\sum_{m=1}^{\ell}\left(\begin{array}{c}
\ell+1 \\
m
\end{array}\right)\left(\partial_{z}^{m} \mu\right) \partial_{a}^{j} \partial_{z}^{\ell+1-m} g^{n+1} \\
-\sum_{m=1}^{j}\left(\begin{array}{c}
j+1 \\
m
\end{array}\right)\left(\partial_{a}^{m}\left(\chi z+r^{n} \chi a\right)\right) \partial_{a}^{j+1-m} \partial_{z}^{\ell} g^{n+1} \\
-\ell \sum_{m=0}^{j+1}\left(\begin{array}{c}
j+1 \\
m
\end{array}\right)\left(\partial_{a}^{m} \chi\right) \partial_{a}^{j+1-m} \partial_{z}^{\ell-1} g^{n+1} \\
+\left[\partial_{a}^{j+1} \partial_{z}^{\ell}\left(g^{n+1} \chi \Theta_{c}\left(y^{n}, f^{n}\right)\right)-\left(\partial_{a}^{j+1} \partial_{z}^{\ell} g^{n+1}\right) \chi \Theta_{c}\left(y^{n}, f^{n}\right)\right] .
\end{gathered}
$$

Using inequalities for Sobolev functions, we have an estimate for $\Phi$, namely

$$
\|\Phi\|_{L^{2}} \leq c\left(1+\left|r^{n}(t)\right|+\left\|\Theta_{c}\left(y^{n}, f^{n}\right)\right\|_{H^{s+1}}\right)\left\|g^{n+1}\right\|_{H^{s}} .
$$

Since $\Theta_{c}$ is smooth and since the prior iterates satisfy (27), (31), and (29), we see that we may bound $\Phi$ by a constant (independent of our parameters 
$n$ and $\delta$ ) times the norm of $g^{n+1}$, i.e.

$$
\|\Phi\|_{L^{2}} \leq c\left\|g^{n+1}\right\|_{H^{s}} .
$$

We proceed by substituting (37) into (36):

$$
\begin{gathered}
\frac{d E_{j, \ell}}{d t}=\int_{D} \frac{\sigma^{2}}{2}\left(\partial_{a}^{j} \partial_{z}^{\ell} g^{n+1}\right)\left(\partial_{a}^{j} \partial_{z}^{\ell+2} g^{n+1}\right) d a d z \\
+\int_{D}(\ell+2) \sigma\left(\partial_{z} \sigma\right)\left(\partial_{a}^{j} \partial_{z}^{\ell} g^{n+1}\right)\left(\partial_{a}^{j} \partial_{z}^{\ell+1} g^{n+1}\right) d a d z \\
\quad-\int_{D} \mu\left(\partial_{a}^{j} \partial_{z}^{\ell} g^{n+1}\right)\left(\partial_{a}^{j} \partial_{z}^{\ell+1} g^{n+1}\right) d a d z \\
-\int_{D}\left(\chi z+r^{n} \chi a\right)\left(\partial_{a}^{j} \partial_{z}^{\ell} g^{n+1}\right)\left(\partial_{a}^{j+1} \partial_{z}^{\ell} g^{n+1}\right) d a d z \\
-\int_{D} \chi \Theta_{c}\left(y^{n}, f^{n}\right)\left(\partial_{a}^{j} \partial_{z}^{\ell} g^{n+1}\right)\left(\partial_{a}^{j+1} \partial_{z}^{\ell} g^{n+1}\right) d a d z+\int_{D} \Phi\left(\partial_{a}^{j} \partial_{z}^{\ell} g^{n+1}\right) d a d z \\
=I+I I+I I I+I V+V+V I .
\end{gathered}
$$

We integrate $I$ by parts with respect to $z$ and add the result to $I I$, finding

$$
\begin{aligned}
I+I I=-\int_{D} \frac{\sigma^{2}}{2} & \left(\partial_{a}^{j} \partial_{z}^{\ell+1} g^{n+1}\right)^{2} d a d z \\
& +(\ell+1) \int_{D} \sigma\left(\partial_{z} \sigma\right)\left(\partial_{a}^{j} \partial_{z}^{\ell} g^{n+1}\right)\left(\partial_{a}^{j} \partial_{z}^{\ell+1} g^{n+1}\right) d a d z,
\end{aligned}
$$

where the properties of $\sigma$ eliminate the presence of a boundary term. The first integral on the right-hand side could be used to find gain of regularity, but we will not need this for the present and we instead simply note that it is nonpositive. The second integral on the right-hand side can be integrated by parts with respect to $z$ once more (and there is again no boundary term), yielding

$$
I+I I \leq-\frac{\ell+1}{2} \int_{D}\left(\sigma \partial_{z}^{2} \sigma+\left(\partial_{z} \sigma\right)^{2}\right)\left(\partial_{a}^{j} \partial_{z}^{\ell} g^{n+1}\right)^{2} d a d z .
$$

There exists $c>0$, then, depending on the function $\sigma$ such that

$$
I+I I \leq c E .
$$

Next, we integrate $I I I$ by parts with respect to the $z$ variable, and we integrate each of $I V$ and $V$ by parts with respect to the $a$ variable. This yields the following:

$$
\begin{gathered}
I I I=\int_{D} \frac{\partial_{z} \mu}{2}\left(\partial_{a}^{j} \partial_{z}^{\ell} g^{n+1}\right)^{2} d a d z \\
I V=\int_{D} \frac{\partial_{a}\left(\chi z+r^{n} \chi a\right)}{2}\left(\partial_{a}^{j} \partial_{z}^{\ell} g^{n+1}\right)^{2} d a d z \\
V=\int_{D} \frac{\partial_{a}\left(\chi \Theta_{c}\left(y^{n}, f^{n}\right)\right)}{2}\left(\partial_{a}^{j} \partial_{z}^{\ell} g^{n+1}\right)^{2} d a d z .
\end{gathered}
$$


Here, there is no boundary term when integrating by parts in $I I I$ because of the properties of $\mu$ at $z_{\min }$ and $z_{\max }$. There are no boundary terms in $I V$ and $V$ when integrating by parts because of the presence of the factors of $\chi$. Just as we bounded $I+I I$ in (39), we may bound $I I I$ :

$$
I I I \leq c E .
$$

For $I V$ and $V$, since they involve the prior iterates, we must utilize the inductive hypothesis. For $I V$, we use (31) to find

$$
I V \leq c\left(1+\frac{2 P_{\max }}{K_{\text {data }}}\right) E .
$$

Since the constants $P_{\max }$ and $K_{d a t a}$ are considered to be fixed (and especially, they do not depend on $n$ or $\delta$ ), we incorporate these into the constant $c$ to write this as

$$
I V \leq c E .
$$

Since the function $\Theta_{c}$ is continuous, there exists a constant $c>0$ such that for all $\tilde{y}$ and $\tilde{f}$ satisfying $\|\tilde{y}\|_{H^{s+1}} \leq M\left\|y_{T}\right\|_{H^{s+1}}$ and $\tilde{f} \in[1 / 2,2]$, we have $\left\|\partial_{a} \Theta_{c}(\tilde{y}, \tilde{f})\right\|_{L^{\infty}(D)} \leq c$. In light of (27) and (29), then, we conclude

$$
V \leq c E \text {. }
$$

Finally, we may use (38) directly to bound $V I$ as

$$
V I \leq c E .
$$

Adding (39), (40), (41), (42), and (43), also summing over $j$ and $\ell$, we have

$$
\frac{d E}{d t} \leq c E
$$

with this constant $c$ independent of $n$ and $\delta$.

Thus, as claimed, for the given value $M>1$ chosen above, there exists $T_{g}>0$ such that if $T \in\left(0, T_{g}\right)$, then for all $t \in[0, T]$,

$$
\left\|g^{n+1}(t, \cdot)\right\|_{H^{s}} \leq M\left\|g_{0}\right\|_{H^{s}},
$$

and this value of $T_{g}$ is independent of both our parameters $n$ and $\delta$.

The details for $y^{n+1}$ are very similar and we omit them. Our conclusion is that there exists $T_{y}>0$ such if $T \in\left(0, T_{y}\right)$, then for all $t \in[0, T]$,

$$
\left\|y^{n+1}(t, \cdot)\right\|_{H^{s+1}} \leq M\left\|y_{T}\right\|_{H^{s+1}} .
$$

Again, this value of $T_{y}$ is independent of $n$ and $\delta$.

We now turn to the estimates for $r^{n+1}, f^{n+1}$, and $K^{n+1}$. The bound for $r^{n+1}$ is immediate from the definition (25), the definition of $P_{\max }$, and the bounds in the inductive hypothesis (27), (28), (29), and (30). Given the bound (31) and the formula (24) for $f^{n+1}$, we see that there exists $T_{f}>0$, independent of $n$ and $\delta$, such that if $T \in\left(0, T_{f}\right)$ then for all $t \in[0, T]$ we have $f^{n+1}(t) \in[1 / 2,2]$.

We next deal with $K^{n+1}$, as defined in (26). Given the bounds on the $n$-th iterates in the inductive hypothesis, we see that for sufficiently small values 
of the time horizon, $g^{n}$ remains close to the initial data $g_{0, \delta}, f^{n}$ remains close to its terminal value which is $f^{n}(T)=1$, and $y^{n}$ remains close to its terminal data $y_{T, \delta}$. We conclude that there exists $T_{K}>0$, with this value independent of $n$ and $\delta$, such that if $T \in\left(0, T_{K}\right)$, then for all $t \in[0, T]$, we have $K^{n+1}(t) \geq K_{\text {data }} / 2$. (To be clear, we have already taken $\delta$ sufficiently small so that the initial iterate $K^{0}$ satisfies $K^{0} \geq 3 K_{\text {data }} / 4$, and the value of $T_{K}$ is otherwise independent of $\delta$.)

Finally we wish to ensure that $y^{n+1}+f^{n+1} w_{\infty}$ remains bounded below by $W / 2$. Similarly to the bound for $K^{n+1}$, the bounds of the inductive hypothesis imply that the time derivatives of $y^{n+1}$ and $f^{n+1}$ are uniformly bounded, and thus if $T$ is sufficiently small, the minimum of $y^{n+1}+f^{n+1} w_{\infty}$ remains close to its terminal value, which by (17) is at least $3 W / 4$. Thus there exists $T_{W}>0$ such that if $T \in\left(0, T_{W}\right)$, then

$$
\min _{(t, a, z) \in[0, T] \times D}\left(y^{n+1}+f^{n+1} w_{\infty}\right) \geq \frac{W}{2},
$$

and this value of $T_{W}$ is independent of $n$ and $\delta$.

Choosing $T_{*}=\min \left\{T_{g}, T_{y}, T_{f}, T_{K}, T_{W}\right\}$, the proof is complete.

\section{Passage to the limit}

We now take the limit of our iterates, proving our main theorem.

Theorem 5. Let $s \in \mathbb{N}$ satisfying $s \geq 4$ be given, and let $w_{\infty}>0$ be given. Let $A>0$ be given and let the spatial domain $D$ be as above. Let $y_{T} \in H^{s+1}(D)$ and $g_{0} \in H^{s}(D)$ be given, such that the support of $g_{0}$ with respect to a and the support of $y_{T}$ with respect to a are in the interior of the interval $[-A, A]$, and assume that $g_{0}$ is a probability measure. Assume $W>0$, where $W$ is defined by (16). There exists $T_{* *}>0$ such that if $T \in\left(0, T_{* *}\right)$, then there exists $y \in L^{\infty}\left([0, T] ; H^{s+1}(D)\right) \cap C\left([0, T] ; H^{s^{\prime}+1}(D)\right)$ for all $s^{\prime}<s$, and $g \in L^{\infty}\left([0, T] ; H^{s}(D)\right) \cap C\left([0, T] ; H^{s^{\prime}}(D)\right)$ for all $s^{\prime}<s$, and $f \in C^{1}([0, T])$, such that $K[y, g, f]>0$ for all $t \in[0, T]$ and with $r$ defined by $r=P[y, g, f] / K[y, g, f]$, then $(y, g, f)$ solve (7), (8), and (6) with data $g(0, \cdot)=g_{0}, y(T, \cdot)=y_{T}$, and $f(T)=1$. The solution $g$ is a probability measure at each time $t \in[0, T]$ and $y+f w_{\infty}$ is positive at each time $t \in[0, T]$.

We make a remark on the data and the constraint $\mathcal{C}=0$ before beginning the proof.

Remark 6. Note that we have not required in our existence theorem that $\iint a g_{0} d a d z=0$; the existence theorem holds whether or not the constraint is initially satisfied. Of course if one hopes to have $\mathcal{C}=0$ for all time, then the initial data should be taken to satisfy $\mathcal{C}(t=0)=0$.

Proof. We have previously suppressed the dependence of the solutions on the mollification parameter $\delta$, and we have left this value $\delta>0$ to be arbitrary. We now consider the sequence of solutions resulting from taking a specific value of $\delta$ for each $n \in \mathbb{N}$, namely $\delta=1 / n$. In this section we will show that 
there is a subsequence of $\left(y^{n}, g^{n}, f^{n}, K^{n}, r^{n}\right)$ which converges to a solution of the transformed system.

We restrict $T$ to values in $\left(0, T_{*}\right)$, with $T_{*}$ given by Theorem 4 . There will be another restriction on $T$ later in the proof.

We begin with $y^{n}$. By Theorem 4, on the time interval $[0, T]$, the sequence $y^{n}$ is uniformly bounded in $H^{s+1}(D)$. With $s \geq 2$, Sobolev embedding then implies that $\nabla_{a, z} y^{n}$ is bounded in $L^{\infty}$ uniformly with respect to $n$. Insepecting the family of evolution equations (21), again using the uniform bounds of Theorem 4 and now using $s \geq 3$, we see that $\partial_{t} y^{n}$ is bounded in $L^{\infty}$, uniformly with respect to $n$. We conclude that $\left\{y^{n}: n \in \mathbb{N}\right\}$ is an equicontinuous family, and we apply the Arzela-Ascoli theorem to find a uniformly convergent subsequence, which we do not relabel. We call the limit $y$.

We now address regularity of the limit. The Arzela-Ascoli theorem gives convergence in $C([0, T] \times D)$, and this immediately implies convergence in $C\left([0, T] ; L^{2}(D)\right)$. With the uniform bound in $H^{s+1}$ from Theorem 4, Sobolev interpolation then implies convergence in $C\left([0, T] ; H^{s^{\prime}+1}\right)$, for any $s^{\prime}<s$. Furthermore, since the iterates are uniformly bounded in $H^{s+1}$, at every time $t \in[0, T]$ there is a weak limit in $H^{s+1}$ obeying the same bound, and this limit must again equal $y$. Thus $y$ is also in $L^{\infty}\left([0, T] ; H^{s+1}\right)$.

The argument for $g^{n}$ is the same, except that $g^{n}$ being bounded in $H^{s}$ rather than $H^{s+1}$ means that we require $s \geq 4$ to have the $L^{\infty}$ bound on the time derivatives. We call the limit of the subsequence (which we do not relabel) $g$. This $g$ is in $C\left([0, T] ; H^{s^{\prime}}\right)$ for any $s^{\prime}<s$, and also is in $L^{\infty}\left([0, T] ; H^{s}\right)$.

We next take the limit of $f^{n}$. From the uniform bounds of Theorem 4 , inspection of (23) implies that $\left(f^{n}\right)^{\prime}$ is uniformly bounded. Thus $\left\{f^{n}: n \in\right.$ $\mathbb{N}\}$ is an equicontinuous family on $[0, T]$. The Arzela-Ascoli theorem again applies, yielding a uniform limit of a subsequence (which we do not relabel); we call the limit $f$.

We turn now to the sequence $K^{n}$. Considering (26) and the fact that $y^{n}$, $g^{n}$, and $f^{n}$ all converge uniformly, we see that $K^{n}$ converges to a limit $K$ which is given by

$$
K=\iint g\left(H_{p p}\left(\psi\left(y+f w_{\infty}\right)\right)\right)\left(y+f w_{\infty}\right) d a d z,
$$

and this convergence is uniform. Since we have $K^{n} \geq K_{d a t a} / 2$ for all $n$, we also have $K \geq K_{\text {data }} / 2$ for all times.

Finally we consider convergence of $r^{n}$. In light of (25) and since we know that $K^{n}$ converges, we only need to consider the convergence of $P^{n}$. The convergence that we have established for $y^{n}$ implies that up through second derivatives of $y^{n}$ converge to the appropriate derivatives of $y$. Similarly, up to second derivatives of $g^{n}$ converge uniformly to the appropriate derivatives of $g$. This is enough regularity to ensure that $P^{n}=P_{c}\left[y^{n}, f^{n}, g^{n}\right]$ converges uniformly to $P_{c}[y, f, g]$. Since $P^{n}$ and $K^{n}$ both converge, we see that $r^{n}$ converges to $r=P / K$, and this convergence is uniform. 
We next demonstrate that the limits $y$ and $g$ satisfy the appropriate equations. We provide the details only for $g$, as the argument for $y$ is the same. We integrate (19) with respect to time, on the interval $[0, t]$ :

$$
\begin{array}{r}
g^{n+1}(t, \cdot)=g_{0,1 / n}+\int_{0}^{t} \frac{1}{2} \partial_{z z}\left(\sigma^{2} g^{n+1}\right)-\partial_{z}\left(\mu g^{n+1}\right)-\partial_{a}\left(\chi\left(z+r^{n} a\right) g^{n+1}\right) \\
-\partial_{a}\left(\chi \Theta_{c}\left(y^{n}, f^{n}\right) g^{n+1}\right) d t^{\prime} .
\end{array}
$$

The uniform convergence of the iterates $y^{n}, g^{n}, f^{n}$, and $r^{n}$ implies convergence of the integral. Taking the limit, we have

$g(t, \cdot)=g_{0}+\int_{0}^{t} \frac{1}{2} \partial_{z z}\left(\sigma^{2} g\right)-\partial_{z}(\mu g)-\partial_{a}(\chi(z+r a) g)-\partial_{a}\left(\chi \Theta_{c}(y, f) g\right) d t^{\prime}$.

Taking the time derivative of this, we see that

$$
\partial_{t} g-\frac{1}{2} \partial_{z z}\left(\sigma^{2} g\right)+\partial_{z}(\mu g)+\partial_{a}(\chi(z+r a) g)+\partial_{a}\left(\chi \Theta_{c}(y, f) g\right)=0 .
$$

Similarly, we conclude that the equation satisfied by $y$ is

$$
\partial_{t} y+\frac{1}{2} \sigma^{2} y+\mu \partial_{z} y+(r-\rho) y+(\chi(z+r a)) \partial_{a} y+\chi \Theta_{c}(y, f) \partial_{a} y=0
$$

The last step in the existence proof is to remove the cutoff functions $\chi$ and $\psi$. As discussed when the iterative scheme was set up, as long as the iterates remain uniformly bounded, and as long as $T$ is small enough, the support of the iterates with respect to the $a$ variable remains within the set $A_{2}$. Since the iterates converge uniformly, the support of $y$ and $g$ with respect to the $a$ variable also remains confined to the set $A_{2}$ throughout the interval $[0, T]$. Since the cutoff function satisfies $\chi=1$ when restricted to $A_{2}$, we see that in (44) and (45), we have $\chi g=g$ and $\chi \partial_{a} y=\partial_{a} y$. Since (32) is satisfied for all $n$ and since $y^{n}$ and $f^{n}$ converge to $y$ and $f$, we see that

$$
\min _{(t, a, z) \in[0, T] \times D}\left(y(t, a, z)+f(t) w_{\infty}\right) \geq \frac{W}{2} .
$$

This implies $\psi\left(y+f w_{\infty}\right)=y+f w_{\infty}$, and therefore that $\Theta_{c}(y, f)=\Theta(y, f)$. We conclude that the equations satisfied by $y$ and $g$ are (7) and (8), as desired.

The proof of the existence theorem is complete.

\section{UNIQUENESS}

We now prove uniqueness of our solutions.

Theorem 7. Let $s \in \mathbb{N}$ satisfying $s \geq 4$ be given, and let $w_{\infty}>0$ be given. Let $A>0$ be given and let the spatial domain $D$ be as above. Let $y_{T} \in H^{s+1}(D)$ and $g_{0} \in H^{s}(D)$ be given, such that the support of $g_{0}$ with respect to a and the support of $y_{T}$ with respect to a are in the interior of the interval $[-A, A]$, and assume that $g_{0}$ is a probability measure. Assume $W>0$, where $W$ is defined by (16). Let $\left(y_{1}, g_{1}, f_{1}\right)$ and $\left(y_{2}, g_{2}, f_{2}\right)$ and the 
associated interest rates $r_{i}=P\left[y_{i}, g_{i}, f_{i}\right] / K\left[y_{i}, g_{i}, f_{i}\right]$ satisfy (7), (8), (6), with data $g_{i}(0, \cdot)=g_{0}, y_{i}(T, \cdot)=y_{T}$, and $f_{i}(T)=1$. Let $T>0$ be such that $y_{i} \in L^{\infty}\left([0, T] ; H^{s+1}(D)\right) \cap C\left([0, T] ; H^{s^{\prime}+1}(D)\right)$, for all $s^{\prime}<s$, and such that $g_{i} \in L^{\infty}\left([0, T] ; H^{s}(D)\right) \cap C\left([0, T] ; H^{s^{\prime}}(D)\right)$, for all $s^{\prime}<s$, and such that $f_{i} \in C^{1}([0, T])$. Assume that $g_{i}$ and $y_{i}$ are compactly supported with respect to the a variable in the interval $(-2 A, 2 A)$. There exists $T_{* * *}$ such that if $T \in\left(0, T_{* * *}\right)$, then $\left(y_{1}, g_{1}, f_{1}\right)=\left(y_{2}, g_{2}, f_{2}\right)$.

Proof. By arguments such as those in [11] we see that the evolution for $\partial_{a} v=y+f w_{\infty}$ is positivity preserving, and thus we do not need to assume an explicit lower bound for $y+f w_{\infty}$ over the given interval $[0, T]$. Similarly we could dispense with the explicit bound on the support with respect to the $a$ variable, but we do state it here so as to keep the domain consistent with the solutions we have already proved to exist.

We define three components for the energy for the difference of two solutions, called $E_{d, g}, E_{d, y}$, and $E_{d, f}$, where

$$
\begin{gathered}
E_{d, g}(t)=\frac{1}{2} \iint\left(g_{1}-g_{2}\right)^{2} d a d z, \\
E_{d, y}(t)=\frac{1}{2} \iint\left|\nabla_{a, z}\left(y_{1}-y_{2}\right)\right|^{2} d a d z,
\end{gathered}
$$

and

$$
E_{d, f}=\sup _{t \in[0, T]} \frac{1}{2}\left|f_{1}(t)-f_{2}(t)\right|^{2}
$$

Note that $E_{d, g}(0)=0$ and that $E_{d, y}(T)=0$.

We start by estimating $E_{d, f}$. Noting that for $i \in\{1,2\}$ we have the equations $f_{i}^{\prime}=\left(\rho-r_{i}\right) f_{i}$, and that $f_{i}(T)=1$, we can write, for any $t \in[0, T]$,

$$
\frac{1}{2}\left|f_{1}(t)-f_{2}(t)\right|^{2}=-\int_{t}^{T}\left(f_{1}\left(t^{\prime}\right)-f_{2}\left(t^{\prime}\right)\right)\left(f_{1}^{\prime}\left(t^{\prime}\right)-f_{2}^{\prime}\left(t^{\prime}\right)\right) d t^{\prime} .
$$

Substituting from the equations for $f_{i}^{\prime}$ and adding and subtracting, this becomes

$$
\frac{1}{2}\left|f_{1}(t)-f_{2}(t)\right|=-\int_{t}^{T}\left(f_{1}-f_{2}\right)\left(r_{2}-r_{1}\right) f_{1} d t^{\prime}-\int_{t}^{T}\left(f_{1}-f_{2}\right)^{2}\left(\rho-r_{2}\right) d t^{\prime} .
$$

Taking the supremum with respect to time and performing some other manipulations, we can bound this as

$$
E_{d, f} \leq c T E_{d, f}+c T\left|r_{1}-r_{2}\right|_{L^{\infty}}^{2}
$$

We will now work with $r_{1}-r_{2}$. Since $r_{i}=P_{i} / K_{i}$, it is clear that at any time $r_{1}-r_{2}$ can be bounded in terms of $K_{1}-K_{2}$ and $P_{1}-P_{2}$. We consider 
$K_{1}-K_{2}$ first. For any $t \in[0, T]$, we have

$$
\begin{aligned}
\left|K_{1}(t)-K_{2}(t)\right|=\mid \iint g_{1}\left(H_{p p}\left(y_{1}+f_{1} w_{\infty}\right)\right)\left(y_{1}+f_{1} w_{\infty}\right) d a d z \\
\quad-\iint g_{2}\left(H_{p p}\left(y_{2}+f_{2} w_{\infty}\right)\left(y_{2}+f_{2} w_{\infty}\right) d a d z \mid .\right.
\end{aligned}
$$

After some adding and subtracting and using a Lipschitz estimate for $H_{p p}$, it is evident that this can be bounded by

$$
\left|K_{1}(t)-K_{2}(t)\right| \leq c\left(E_{g}^{1 / 2}+E_{y}^{1 / 2}+E_{f}^{1 / 2}\right) .
$$

We will need (47) as well as the supremum of this with respect to time,

$$
\left|K_{1}-K_{2}\right|_{L^{\infty}} \leq c\left(\sup _{t \in[0, T]}\left(E_{g}^{1 / 2}+E_{y}^{1 / 2}\right)+E_{f}^{1 / 2}\right) .
$$

The difference $P_{1}-P_{2}$ is similar but slightly more involved, as we must integrate by parts in some instances. We start by noting that the definition (8) of $P$ includes three terms, so we decompose $P_{1}-P_{2}$ as

$$
P_{1}-P_{2}=\Upsilon_{I}+\Upsilon_{I I}+\Upsilon_{I I I} .
$$

We believe the meaning here is clear, and we will only write out $\Upsilon_{I I}$ in detail. We add and subtract to decompose $\Upsilon_{I I}$ as

$$
\Upsilon_{I I}=\sum_{j=1}^{7} \Upsilon_{I I, j}
$$

where we have the following definitions:

$$
\begin{aligned}
& \Upsilon_{I I, 1}=\iint\left(g_{1}-g_{2}\right) H_{p p}\left(y_{1}+f_{1} w_{\infty}\right) \cdot \\
& \cdot\left(-\frac{1}{2} \sigma^{2} \partial_{z z} y_{1}-\mu \partial_{z} y_{1}-H_{p}\left(y_{1}+f_{1} w_{\infty}\right) \partial_{a} y_{1}+\rho\left(y_{1}+f_{1} w_{\infty}\right)\right) d a d z \\
& \Upsilon_{I I, 2}=\iint g_{2}\left[H_{p p}\left(y_{1}+f_{1} w_{\infty}\right)-H_{p p}\left(y_{2}+f_{2} w_{\infty}\right)\right] \cdot \\
& \cdot\left(-\frac{1}{2} \sigma^{2} \partial_{z z} y_{1}-\mu \partial_{z} y_{1}-H_{p}\left(y_{1}+f_{1} w_{\infty}\right) \partial_{a} y_{1}+\rho\left(y_{1}+f_{1} w_{\infty}\right)\right) d a d z \\
& \Upsilon_{I I, 3}=\iint g_{2} H_{p p}\left(y_{2}+f_{2} w_{\infty}\right)\left(-\frac{1}{2} \sigma^{2} \partial_{z z}\left(y_{1}-y_{2}\right)\right) d a d z \\
& \Upsilon_{I I, 4}=\iint g_{2} H_{p p}\left(y_{2}+f_{2} w_{\infty}\right)\left(-\mu \partial_{z}\left(y_{1}-y_{2}\right)\right) d a d z, \\
& \Upsilon_{I I, 5}=\iint g_{2} H_{p p}\left(y_{2}+f_{2} w_{\infty}\right)\left(-H_{p}\left(y_{1}+f_{1} w_{\infty}\right)+H_{p}\left(y_{2}+f_{2} w_{\infty}\right)\right) \partial_{a} y_{1} d a d z
\end{aligned}
$$




$$
\Upsilon_{I I, 6}=\iint g_{2} H_{p p}\left(y_{2}+f_{2} w_{\infty}\right)\left(-H_{p}\left(y_{2}+f_{2} w_{\infty}\right) \partial_{a}\left(y_{1}-y_{2}\right)\right) d a d z
$$

and

$$
\Upsilon_{I I, 7}=\iint g_{2} H_{p p}\left(y_{2}+f_{2} w_{\infty}\right) \rho\left(y_{1}-y_{2}+\left(f_{1}-f_{2}\right) w_{\infty}\right) d a d z .
$$

It is immediate that $\Upsilon_{I I, 1}, \Upsilon_{I I, 2}, \Upsilon_{I I, 5}$, and $\Upsilon_{I I, 7}$ may be bounded in terms of $E_{d, g}, E_{d, y}$, and $E_{d, f}$; note that Lipschitz estimates for $H_{p}$ and $H_{p p}$ are needed, but these are smooth functions and the Lipschitz estimates are thus available. The terms $\Upsilon_{I I, 3}$ and $\Upsilon_{I I, 4}$ may be bounded in terms of $E_{d, y}$ after integrating by parts with respect to the $z$ variable; note that there are no boundary terms because of the properties of the diffusion and transport coefficients, $\sigma$ and $\mu$, at the boundaries of the domain. The term $\Upsilon_{I I, 6}$ can be bounded in terms of $E_{d, y}$ after an integration by parts with respect to the $a$ variable; there is no boundary term because solutions are compactly supported with respect to the $a$ variable. We omit further details, and the result of these and similar considerations is the bounds

$$
\left|P_{1}(t)-P_{2}(t)\right| \leq c\left(E_{g}^{1 / 2}+E_{y}^{1 / 2}+E_{f}^{1 / 2}\right),
$$

and

$$
\left|P_{1}-P_{2}\right|_{L^{\infty}} \leq c\left(\sup _{t \in[0, T]}\left(E_{g}^{1 / 2}+E_{y}^{1 / 2}\right)+E_{f}^{1 / 2}\right) .
$$

From our bounds on differences of $K$ and $P$, we conclude that at each $t \in[0, T]$

$$
\left|r_{1}(t)-r_{2}(t)\right| \leq c\left(E_{g}^{1 / 2}+E_{y}^{1 / 2}+E_{f}^{1 / 2}\right),
$$

and taking the supremum in time,

$$
\left|r_{1}(t)-r_{2}(t)\right|_{L^{\infty}} \leq c\left(\sup _{t \in[0, T]}\left(E_{g}^{1 / 2}+E_{y}^{1 / 2}\right)+E_{f}^{1 / 2}\right) .
$$

Using this in (46), our bound for $E_{d, f}$ is

$$
E_{d, f} \leq c T\left(\sup _{t \in[0, T]}\left(E_{d, g}+E_{d, y}\right)+E_{d, f}\right) .
$$

We next establish that there exists $c>0$ such that

$$
\frac{d E_{d, g}}{d t} \leq c\left(E_{d, g}+E_{d, y}+E_{d, f}\right)
$$

and

$$
\frac{d E_{d, y}}{d t} \leq c\left(E_{d, g}+E_{d, y}+E_{d, f}\right) .
$$

To this end, we take the time derivative of $E_{d, g}$ :

$$
\frac{d E_{d, g}}{d t}=\iint\left(g_{1}-g_{2}\right) \partial_{t}\left(g_{1}-g_{2}\right) d a d z .
$$


We then substitute from the equations (8) satisfied by each $g_{i}$, and add and subtract:

$$
\begin{gathered}
\frac{d E_{d, g}}{d t}=\frac{1}{2} \iint\left(g_{1}-g_{2}\right) \partial_{z z}\left(\sigma^{2}\left(g_{1}-g_{2}\right)\right) d a d z \\
-\iint\left(g_{1}-g_{2}\right) \partial_{z}\left(\mu\left(g_{1}-g_{2}\right)\right) d a d z-\iint\left(g_{1}-g_{2}\right) \partial_{a}\left(\left(r_{1}-r_{2}\right) a g_{1}\right) d a d z \\
-\iint\left(g_{1}-g_{2}\right) \partial_{a}\left(\left(z+r_{2} a\right)\left(g_{1}-g_{2}\right)\right) d a d z \\
-\iint\left(g_{1}-g_{2}\right) \partial_{a}\left(\left(g_{1}-g_{2}\right) \Theta\left(y_{1}, f_{1}\right)\right) d a d z \\
-\iint\left(g_{1}-g_{2}\right) \partial_{a}\left(g_{2}\left(\Theta\left(y_{1}, f_{1}\right)-\Theta\left(y_{2}, f_{2}\right)\right)\right) d a d z
\end{gathered}
$$

There are six terms on the right-hand side, and estimating these is very much like the estimate for $g^{n+1}$ in the proof of Theorem 4. Specifically, for the first term, the two derivatives with respect to $z$ should be applied, and then some integrations by parts can be made. For the second term, the one derivative with respect to $z$ can be applied, and then an integration by parts can be made. To estimate the third term, the bound (49) is employed. For the fourth and fifth terms, the derivative with respect to $a$ should be applied, and then an integration by parts can be made. For the sixth term, the derivative with respect to $a$ should be applied, a Lipschitz estimate for $\Theta$ is used, and a further addition and subtraction can be utilized as well. We omit further details.

Integrating (51) forward in time, and using the initial data, we find

$$
\begin{aligned}
E_{d, g}(t) \leq c \int_{0}^{t} E_{d, g}\left(t^{\prime}\right)+ & E_{d, y}\left(t^{\prime}\right)+E_{d, f} d t^{\prime} \\
& \leq c T\left(\sup _{t \in[0, T]}\left(E_{d, g}(t)+E_{d, y}(t)\right)+E_{d, f}\right) .
\end{aligned}
$$

Integrating (52) backward in time, and using the terminal data, we find

$$
\begin{aligned}
E_{d, y}(t) \leq c \int_{t}^{T} E_{d, g}\left(t^{\prime}\right)+ & E_{d, y}\left(t^{\prime}\right)+E_{d, f} d t^{\prime} \\
& \leq c T\left(\sup _{t \in[0, T]}\left(E_{d, g}(t)+E_{d, y}(t)\right)+E_{d, f}\right) .
\end{aligned}
$$

Adding (50), (53), and (54), and taking the supremum in time and reorganizing terms, we find

$$
(1-c T)\left(E_{d, f}+\sup _{t \in[0, T]}\left(E_{d, g}(t)+E_{d, y}(t)\right)\right) \leq 0 .
$$

Thus as long as $0<T<1 / c$, we have $y_{1}=y_{2}, g_{1}=g_{2}$, and $f_{1}=f_{2}$. 


\section{Discussion}

We mentioned above that we would remark again on the difference between choice of spatial domain here as compared to 1 . We have taken the same domain with respect to the $z$ variable, but in [1 the domain with respect to the $a$ variable was taken to be $\left[a_{\min }, \infty\right)$ for a given $a_{\min }<0$. We have instead taken the initial support of our functions with respect to the $a$ variable in $[-A, A]$ for a given $A>0$ and the support of our solutions has remained in $[-2 A, 2 A]$ over the time interval $[0, T]$. If we take the view that $a_{\text {min }}<-2 A$, then our solutions fit into the framework of [1] with regard to this aspect.

We also mentioned above that we would comment on our assumption that the range of $u^{\prime}$ is equal to $(0, \infty)$, as would be the case, for instance, if $u(c)=\sqrt{c}$. This assumption is only for simplicity and the general case can be treated by our same method. We stated in Section 2 that the quantity in the definition of the Hamiltonian is maximized when $p=u^{\prime}(c)$, and so $c=\left(u^{\prime}\right)^{-1}(p)$. This formula is still valid if $p$ is in Range $\left(u^{\prime}\right)$, which is necessarily an interval. Thus the formula we have used throughout the work is valid for values of $p$ in a given interval. But $p$ stands in for $\partial_{a} v$, and the method we have applied does find solutions where $\partial_{a} v=y+f w_{\infty}$ only takes values in a given interval. For a general utility function, the terminal data $y_{T}+w_{\infty}$ can be taken with values in the appropriate interval, and the time horizon $T$ can be taken sufficiently small so that solutions remain in this interval.

We have discussed in the introduction that we can show that in some cases, the solutions we have proved to exist are not solutions of the original system. For every solution we have proved to exist via Theorem 5 , there is associated a value of the constant $\mathcal{Q}$. If this constant $\mathcal{Q}$ is nonzero, then the solution does not solve the original problem, i.e., if $\mathcal{Q} \neq 0$, then $\mathcal{C} \not \equiv 0$. It is straightforward to see that we can guarantee in some cases that $\mathcal{Q} \neq 0$. We define

$$
\mathcal{Q}_{\text {data }}=\iint\left(z+H_{p}\left(y_{T}+w_{\infty}\right)\right) g_{0} d a d z
$$

Assume that $g_{0}, y_{T}$, and $w_{\infty}$ are specified such that $\mathcal{Q}_{\text {data }} \neq 0$. Then for sufficiently small values of $T>0$, for the solutions $(y, g, f)$ proved to exist in our main theorem, the solution will not vary much from the data. Therefore for small values of $T$, we will have $\mathcal{Q}$ close to $\mathcal{Q}_{\text {data }}$, and $\mathcal{Q}$ will therefore be nonzero.

As mentioned in the introduction, the authors of [1] proposed a restriction on the choice of terminal values for $v$, and thus in our case for the terminal data for $\partial_{a} v$, which is $y_{T}+w_{\infty}$. In particular they proposed that $T$ should be taken to be fairly large and the final value of $v$ should be associated to a stationary solution. Since a stationary solution can be viewed as the infinite- $T$ limit of solutions of the system under consideration, and stationary solutions would satisfy $\mathcal{C}=0$, this proposed data would be expected to 
yield solutions satisfying only $\mathcal{C} \approx 0$. Further work is warranted, though, to find solutions which satisfy the constraint exactly. Specifically, given a value of the time horizon $T$ and the initial distribution $g_{0}$ initially satisfying the constraint, the author intends to perform computational and analytical studies seeking existence of terminal data $y_{T}+w_{\infty}$ which yield $\mathcal{Q}=\mathcal{C}=0$.

\section{ACKNOWLEDGMENTS}

The author gratefully acknowledges support from the National Science Foundation through grant DMS-1515849.

\section{CONFLiCT OF INTEREST}

Conflict of Interest: The authors declare that they have no conflict of interest.

\section{REFERENCES}

[1] Y. Achdou, F.J. Buera, J.-M. Lasry, P.-L. Lions, and B. Moll. Partial differential equation models in macroeconomics. Philos. Trans. R. Soc. Lond. Ser. A Math. Phys. Eng. Sci., 372(2028):20130397, 19, 2014.

[2] R.A. Adams and J.J.F. Fournier. Sobolev spaces, volume 140 of Pure and Applied Mathematics (Amsterdam). Elsevier/Academic Press, Amsterdam, second edition, 2003.

[3] S.R. Aiyagari. Uninsured idiosyncratic risk and aggregate saving. The Quarterly Journal of Economics, 109(3):659-684, 1994.

[4] D.M. Ambrose. Well-posedness of vortex sheets with surface tension. SIAM J. Math. Anal., 35(1):211-244 (electronic), 2003.

[5] D.M. Ambrose. Small strong solutions for time-dependent mean field games with local coupling. C.R. Acad. Sci. Paris, 354:589-594, 2016.

[6] D.M. Ambrose. Strong solutions for time-dependent mean field games with nonseparable Hamiltonians. J. Math. Pures Appl., 2016. Accepted.

[7] D.M. Ambrose. Existence theory for non-separable mean field games in Sobolev spaces. 2018. Preprint. arXiv:1807.02223. Submitted to Math. Ann.

[8] T. Bewley. Stationary monetary equilibrium with a continuum of independently fluctuating consumers. Contributions to mathematical economics in honor of Gérard Debreu, 79, 1986.

[9] P. Cardaliaguet, P.J. Graber, A. Porretta, and D. Tonon. Second order mean field games with degenerate diffusion and local coupling. NoDEA Nonlinear Differential Equations Appl., 22(5):1287-1317, 2015.

[10] M. Cirant, R. Gianni, and P. Mannucci. Short time existence for a general backward-forward parabolic system arising from mean-field games. 2018. Preprint. arXiv:1806.08138.

[11] A. Constantin and J. Escher. Wave breaking for nonlinear nonlocal shallow water equations. Acta Math., 181(2):229-243, 1998.

[12] W. Feller. On boundaries and lateral conditions for the Kolmogorov differential equations. Ann. of Math. (2), 65:527-570, 1957.

[13] D.A. Gomes, E. Pimental, and H. Sánchez-Morgado. Time-dependent mean-field games in the superquadratic case. 2014. arXiv:1311.6684v3.

[14] D.A. Gomes and E. Pimentel. Time-dependent mean-field games with logarithmic nonlinearities. SIAM J. Math. Anal., 47(5):3798-3812, 2015. 
[15] D.A. Gomes, E.A. Pimentel, and H. Sánchez-Morgado. Time-dependent mean-field games in the subquadratic case. Comm. Partial Diff. Equations, 40:40-76, 2015.

[16] M. Huang, P.E. Caines, and R.P. Malhamé. Large-population cost-coupled LQG problems with nonuniform agents: individual-mass behavior and decentralized $\epsilon$-Nash equilibria. IEEE Trans. Automat. Control, 52(9):1560-1571, 2007.

[17] M. Huang, R.P. Malhamé, and P.E. Caines. Large population stochastic dynamic games: closed-loop McKean-Vlasov systems and the Nash certainty equivalence principle. Commun. Inf. Syst., 6(3):221-251, 2006.

[18] M. Huggett. The risk-free rate in heterogeneous-agent incomplete-insurance economies. Journal of Economic Dynamics and Control, 17(5-6):953-969, 1993.

[19] J.-M. Lasry and P.-L. Lions. Jeux à champ moyen. I. Le cas stationnaire. C. R. Math. Acad. Sci. Paris, 343(9):619-625, 2006.

[20] J.-M. Lasry and P.-L. Lions. Jeux à champ moyen. II. Horizon fini et contrôle optimal. C. R. Math. Acad. Sci. Paris, 343(10):679-684, 2006.

[21] J.-M. Lasry and P.-L. Lions. Mean field games. Jpn. J. Math., 2(1):229-260, 2007.

[22] A.J. Majda and A.L. Bertozzi. Vorticity and incompressible flow, volume 27 of Cambridge Texts in Applied Mathematics. Cambridge University Press, Cambridge, 2002.

[23] R.E. Miller. Extension theorems for homogenization on lattice structures. Appl. Math. Lett., 5(6):73-78, 1992.

[24] A. Porretta. On the planning problem for a class of mean field games. C. R. Math. Acad. Sci. Paris, 351(11-12):457-462, 2013.

[25] A. Porretta. On the planning problem for the mean field games system. Dyn. Games Appl., 4(2):231-256, 2014.

[26] A. Porretta. Weak solutions to Fokker-Planck equations and mean field games. Arch. Ration. Mech. Anal., 216(1):1-62, 2015.

3141 Chestnut St., Department of Mathematics, Drexel University, PhiladelPHIA, PA 19104 USA

E-mail address: dma68@drexel.edu 